Available online at GSC Online Press Directory

GSC Biological and Pharmaceutical Sciences

e-ISSN: 2581-3250, CODEN (USA): GBPSC2

Journal homepage: https://www.gsconlinepress.com/journals/gscbps

(REVIEW ARTICLE)

\title{
Role of nanocomposites in drug delivery
}

\author{
Paravastu Venkata Kamala Kumari *, Yarraguntla Srinivasa Rao and Suvvari Akhila \\ Vignan Institute of Pharmaceutical Technology Duvvada, Visakhapatnam.
}

Publication history: Received on 08 August 2019; revised on 09 September 2019; accepted on 13 September 2019

Article DOI: https://doi.org/10.30574/gscbps.2019.8.3.0150

\begin{abstract}
Nanocomposites have become a promising area of research and development. Nanocomposites are of two types: polymer nanocomposites and clay nanocomposites. In a large field of nanotechnology the polymer nanocomposites which can increase the mechanical properties have become a prominent area of current research and development. The clay nanocomposites increase the additional properties and have dominated the polymer literature. But there are a large number of other significant areas of current and emerging interest. The nanocomposites can be used as drug carriers due to their surface and rheological properties. This review will detail the technology involved with claypolymer based nanocomposites and also include other important areas including barrier properties, flammability resistance, biomedical applications, electrical/ electronic applications and fuel cell interest.
\end{abstract}

Keywords: Nanocomposites; Polymer; Clay; Biomedical

\section{Introduction}

Nanocomposite is a multi-phase solid material where one of the phases has one, two or three dimensions less than $100 \mathrm{~nm}$. The nanocomposite material is a novel product having nano fillers dispersed in the matrix. The structure of a nano-composite is a matrix- filler combination where the fillers like particles, fibers or fragments surrounds and binds together as discrete units in the matrix. Significant efforts are under way to control the nano structure. The properties of nanocomposite materials depend not only on the improvement in their physical properties but also can undergo the unprecedented flexibility and morphology and interfacial characteristics. Most nanocomposites, which are of proven technological importance, are composed of two phases.

These are classified into three types:

- Nano layered composites: composed of alternating layers of nano scale dimension.

- Nano filamentary composites: composed of matrices with embedded nano scale diameter filaments.

- Nano particulate composites: composed of matrices with embedded nano scale particles.

It may also include porous, media, colloids and gels and copolymers. That means it forms a solid combination of bulk matrix and nano dimensional phases that may differ in their properties.

The mechanical, electrical, thermal, optical, electrochemical, properties may differ based on their component of the material. Size limits as shown in Table 1 for these effects can differ such as [1]

\footnotetext{
${ }^{*}$ Corresponding author

E-mail address: kamalaparavastu@gmail.com
} 
Table 1 Size limit of various physical properties

\begin{tabular}{lll}
\hline Sr. No. & Property & Size $(\mathbf{n m})$ \\
\hline 1. & Catalytic & less than $5 \mathrm{~nm}$ \\
2. & Refractive index & less than $50 \mathrm{~nm}$ \\
3. & Magnetic & less than $20 \mathrm{~nm}$ \\
4. & Super para magnetism & less than $100 \mathrm{~nm}$ \\
\hline
\end{tabular}

Nanocomposites can encompass a wide range of materials right from the three dimensional metal matrix composites, two dimensional lamellar composites and nano- wires of single dimension to zero - dimensional core - shells, all representing many variations of nano- mixed and layered materials (Table 2).

The physical, chemical and biological properties of nano materials differ from the properties of individual atoms and molecules or bulk matter. It is possible to control the fundamental properties of materials, such as their melting temperature, magnetic properties, charge capacity and even their color without changing the materials and their chemical compositions.

Table 2 Range of materials encompassed in nanocomposites

\begin{tabular}{lll}
\hline Sr. No. & Types of composites & Inorganic components \\
\hline 1. & Three dimensional framework & Zeolites. \\
2. & Two dimensional layered materials & Clays, metal oxides, metal phosphates, chalcogenides. \\
3. & One and zero dimensional materials & $\left(\mathrm{Mo}_{3} \mathrm{Se}_{3}-\right)_{\mathrm{n}}$ chains and clusters. \\
\hline
\end{tabular}

Inorganic layered materials exist in many varieties. They possess well defined, ordered intra lamellar space potentially accessible by foreign species. This ability enables them to act as matrices for polymers yielding hybrid nanocomposites.

Lamellar nanocomposites can be divided into two distinct classes viz. intercalated and exfoliated. The area of the interface between the matrix and reinforcement phases is typically an order of magnitude greater than for conventional composite materials [2].

\subsection{Advantages of nanocomposites [3]}

- Highly dispersible in aqueous medium.

- Uniform distribution of the active agent over an extended period of time.

- Controlled release of the drug.

- Reduces frequency of administration.

- Augmented stability.

- Penetrate regions inaccessible to other delivery systems.

\subsection{Applications}

- Thin film capacitors for computer chips.

- Solid polymer electrolytes for batteries.

- Automotive engine parts and fuel tanks.

- Impellers and blades.

- Oxygen and gas barriers.

- Food packaging. 


\subsection{Future of Nanocomposites}

The number of applications of nanocomposites has been growing at a rapid rate. It has been reported that in less than two years, the worldwide production is estimated to exceed 600,000 tones and is set to cover the following key areas in the next five to ten years:

- Drug delivery systems

- Anti-corrosion barrier coatings

- UV protection gels

- Lubricants and scratch free paints

- New fire retardant materials

- New scratch/abrasion resistant materials

- Superior strength fibres and films.

The improvement of the mechanical property they have been results an major interest in nanocomposites materials in numerous automotive and general/industrial applications. This may also include the utilization on various vehicle types, door handles, engine covers and intake manifolds and timing belt covers. It also on the include usage of impellers and blades for vacuum cleaners, power housings, mower hoods and covers for portable electronic equipment such as mobile phones, pagers etc.

\section{Nanocomposites - basic ingredients}

There are many commercially available nano- particles that are incorporated into the polymer matrix to form polymer nanocomposites [4]. The selection of a particle is very essential to ensure effective penetration of polymer into the interlayer of desired exfoliated or intercalated product.

Polymer nanocomposites consist of polymeric materials with reinforcement of nano- particles. Polymers can be incorporate into the polymeric species itself. Most commonly used nano- particles include:

- Montmorillonite organo clays (MMT)

- Carbon nano fibers

- Polyhedral oligomericsilsesquioxane (POSS)

- Carbon nano tubes

- Nanosilica (N- silica)

- Nanoaluminium oxide $\left(\mathrm{Al}_{2} \mathrm{O}_{3}\right)$

- Nanotitanium oxide $\left(\mathrm{TiO}_{2}\right)$

There are two main challenges in developing nanocomposite materials after desired polymer has been attained. The choice of nanoparticles requires an interfacial interaction within the polymer matrix.

There should be a proper uniform dispersion and distribution of nano particles within the polymer matrix [5].

\section{Clay- based nanocomposites}

Clays are commonly used in the pharmaceutical industry as excipients or active substances. Several studies are related with the decrease in the oral absorption of numerous medicines by the co- administration with clays, but such interactions may also be used to reach technological and biopharmaceutical advantages. This was the starting point in the use of clays in systems of modified drug release [6, 7].

Natural clay minerals are suitable to be used in modified drug delivery system [8] because due to colloidal particle size, crystalline structure, high specific surface area, charge higher cation exchange capacity and swelling capacity. They can be incorporated into a polymeric host carrier, in order to control the diffusion rate of a dispersed slowrelease material. A nanoclay particle reduces the porosity of the polymer or it get obstructs the diffusion of the active material being released, and increases the path of the diffusion [4]. Clays are inexpensive materials, which can be modified by ion exchange, metal/metal complex impregnation, pillaring and acid treatment to develop catalysts with desired functionally [9]. 
Organically modified layered- silicates or nanoclay have become an attractive class of organic- inorganic hybrid materials because of their potential use in wide range of applications such as in polymer nanocomposites, such as paints,cosmetics, greases, ink, toxic gases, and drug delivery carrier.

\subsection{Clays and their modification}

The clays used for the preparation of nanoclay belong to smectite group clays which are also known as phyllosilicates, the most common of which are:

- Montmorillonite

- Hectorite

- Beidillite

- Nontronite

- Saponite

\subsubsection{Ideal characteristics of clay}

Ideal characteristics of the clay are as follows:

- The clay having an high aspect ratio in the range of 300:1 to 1,500:1 range

- The clay having a platy structure.

- The clay having thickness of less than one nanometer.

- Length band width of the choice clays are in micron range.

- Surface area of the exfoliated platelets usually in the range of $700 \mathrm{~m}^{2} / \mathrm{gram}$.

- Molecular weight of the clay platelets is about $1.3 \times 10^{8}$ Dalton $[4,10]$.

\subsubsection{Montmorillonite clay}

Montmorillonite clay (MMT) belonging from the smectite group composed of silica tetrahedral sheets layered between alumina octahedral sheets as shown in Figure 1[11]. The imperfection of the crystal lattice and that induces a net negative charge that leads to the adsorption of alkaline earth metal ions in the interlayer space. MMT also having free hydroxyl end- groups on the surfaces $[12,13]$. It is used for preparation of nanocomposites, due to the large specific surface area that exhibits good absorbance ability, high cation exchange capacity, standout adhesiveness and drug- carrying capability. Also MMT is a common ingredient in pharmaceutical products, both as excipient and as active support [14]. For the preparation of organic - inorganic hybrids, intercalation of organic species into layered inorganic materials provides not only useful but also convenient route that combine the properties of both the organic host and the organic guest [15].

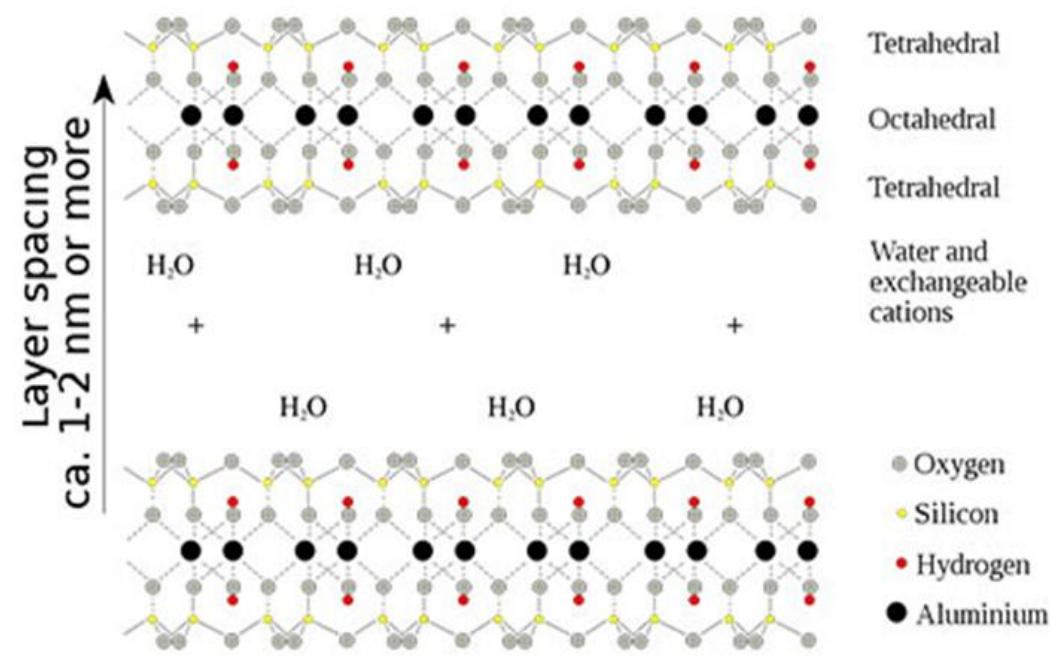

Figure 1 Structure of Montmorillonite clay 
The most commonly used clay in the synthesis of polymer nanocomposites is MMT which is the major constituent of bentonite $[16,17]$. It is well known that filler anisotropy, i.e., large length to diameter ratio is especially favorable in matrix reinforcement. Due to unique structure of MMT, the mineral platelet thickness is only one nanometer, although its dimension in length and width can be measured in hundreds of nanometers, with 200-400 nm range after purification.

One important consequence of the charged nature of the clay is that they are generally high hydrophilic species and therefore, naturally incompatible with wide range of non- polar systems. Organophilic clay can be obtained by simply the ion- exchange reaction of hydrophilic clay with an organic cation such as an alkyl ammonium or phosphonium ion. The inorganic ions, relatively small are exchanged with more voluminous organic oniumcations.

This ion- exchange reaction has two consequences:

- The gap between the single sheets is widened, enabling organic cations chain to move in between them.

- The surface properties of each single sheet are changed from being hydrophilic to hydrophobic or organophilic as shown in Figure 2.
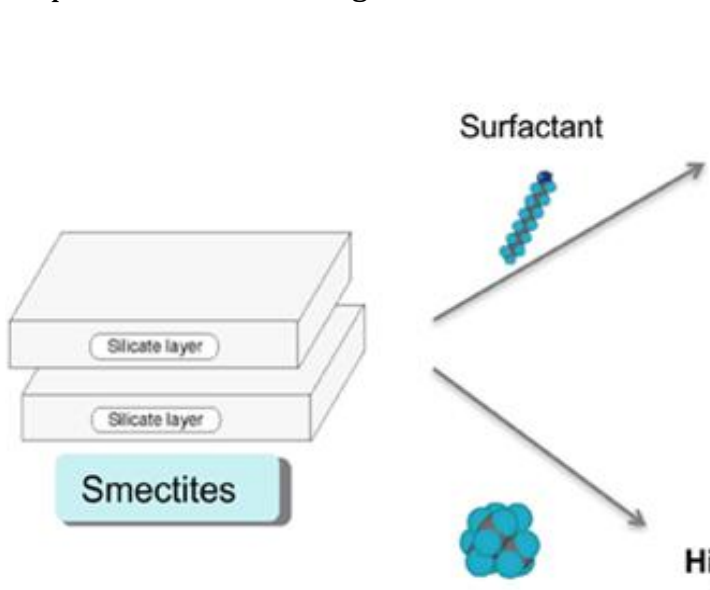

Tetramethylammonium etc.

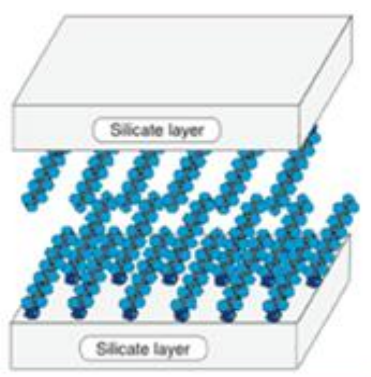

Hydrophobic Smectites

(Amount/Density of Cations)

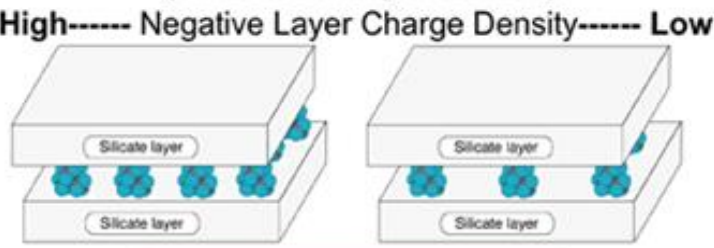

Pillared Smectites

(A recent review) J. Nanosci. Nanotech., 14, 2121-2134 (2014).

Figure 2 Schematic of an ion- exchange reaction in layered silicates

\subsubsection{Mechanisms of clay-drug interactions}

Clay minerals are naturally occurring inorganic cationic exchangers and therefore they may undergo ion exchange with basic drugs in solution. Smectites, especially MMT and saponite, have been the more commonly studied, as compared to other pharmaceutical silicates due to their higher cationic exchange capacity [4].

The major mechanisms of drug intercalation into the montmorillonite are:

- Drug adsorbed onto the free surface of the montmorillonite.

- Drug replaced sodium in the interlayer.

- Drug replaces clay- $\mathrm{OH}$ groups to form ionic bonds with $\mathrm{Al}^{3+}$ and $\mathrm{Mg}^{2+}$ in the montmorillonite.

The first two mechanisms are presumed as the major mechanisms for drug absorbed on to the montmorillinite by a concentration gradient. When the concentration gradient is equal between inside and outside of the montmorillonite, after soaking for a period of time the diffusion would stop [10]. In biological fluids, counterions can displace the drug from the substrate and deliver into the body, while the exchanger is being eliminated. 


\subsubsection{In vivo drug release mechanism}

When prepared nanocomposite is administered in body; biological counterions get exchanged with cationic drug particles which are present on the surface of nanoclay. Free drug molecules are available for absorption and unloaded particle which are having submicron size passes the intestinal membrane and goes in blood stream. Through the kidney unloaded particles excreted out [4].

\subsubsection{Purification of Montmorillonite}

The purity of the clay can affect the final nanocomposite properties; due to this it is very important to have montmorillonite with minimum impurities of crystalline and amorphous silica, calcite, kaolin etc. The technique mainly used for purification of clays includes hydrocyclone, centrifugation, sedimentation method and chemical treatment.

The purified montmorillonite was obtained by dispersing bentonite in $0.1 \mathrm{M} \mathrm{NaCl}$ solution and stirred for $12 \mathrm{hrs}$. The dispersion was reacted three times with $0.1 \mathrm{M} \mathrm{NaCl}$ solution. After centrifugation, the sodium rich bentonite was washed with de- ionized water until free of chloride ion as tested by $\mathrm{AgNO}_{3}$ solution. The MMT dispersion was dried at $90-100^{\circ} \mathrm{C}$ and ground to pass through the 200 mesh sieve. The Cation Exchange Capacity (CEC) of MMT was measured by the standard ammonium acetate method $[18,19]$.

\subsubsection{Characterization of Montmorillonite}

Characterization of clays and their modified organic derivatives are carried out by using simple as well as modern characterization tools which includes determination of chemical compositions by gravimetric analysis, Inductively Coupled Plasma (ICP), Cation Exchange Capacity (CEC), using standard ammonium acetate method, surface area measurement, Scanning Electron Microscopy (SEM), Fourier Transform Infra-Red Spectroscopy (FT-IR), Powdered Xray diffraction (PXRD) and others. Generally, ionic formula is composed on the basis of its chemical compositions, charge density and cationic exchange capacity of clays which provide information about the types of layered silicates. Principally, FT-IR and PXRD are basic instrumental techniques used for identification of clay structure [10, 20].

\section{Polymer nanocomposites}

Polymer nanocomposites are the materials in which nanoscopic inorganic particles, typically 10-100 $\mathrm{A}^{0}$. These are a new alternative to conventionally filled polymers. This may improve the outstanding barrier properties, solvent and heat resistant and decrease the flammability [21].

The alkyl ammonium cations are used in the organoclays to lower the surface energy of the inorganic host and increases the wetting property of a polymer. The alkyl ammonium or phosphonium cations having the functional groups that can react with the polymer and this improve the strength of the interface between the inorganic and the polymer.

Three methods have been developed to produce polymer/ layered silicate composites:

\subsection{In situ polymerization}

In this method a polymer precursor or monomer are inserted in between clay layers and then expanding the layered silicate platelets into the matrix by polymerization. This method has the advantage of producing well- exfoliated nanocomposites and has been applied to a wide range of polymeric systems.

\subsection{Solution- induced intercalation method}

In this method solvents are used to swell and disperse clays into a polymer solution.

\subsection{Melt processing method}

This method applies intercalation and exfoliation of layered silicates in polymeric matrices during melt.

In addition to these the three major processing methods have been developed. These include solid- intercalation covulcanization and the sol- gel method. 
Polymers layered are three types:

- Conventional composites.

- Intercalated nanocomposites.

- Exfoliated nanocomposites as shown in Figure 3 [10]

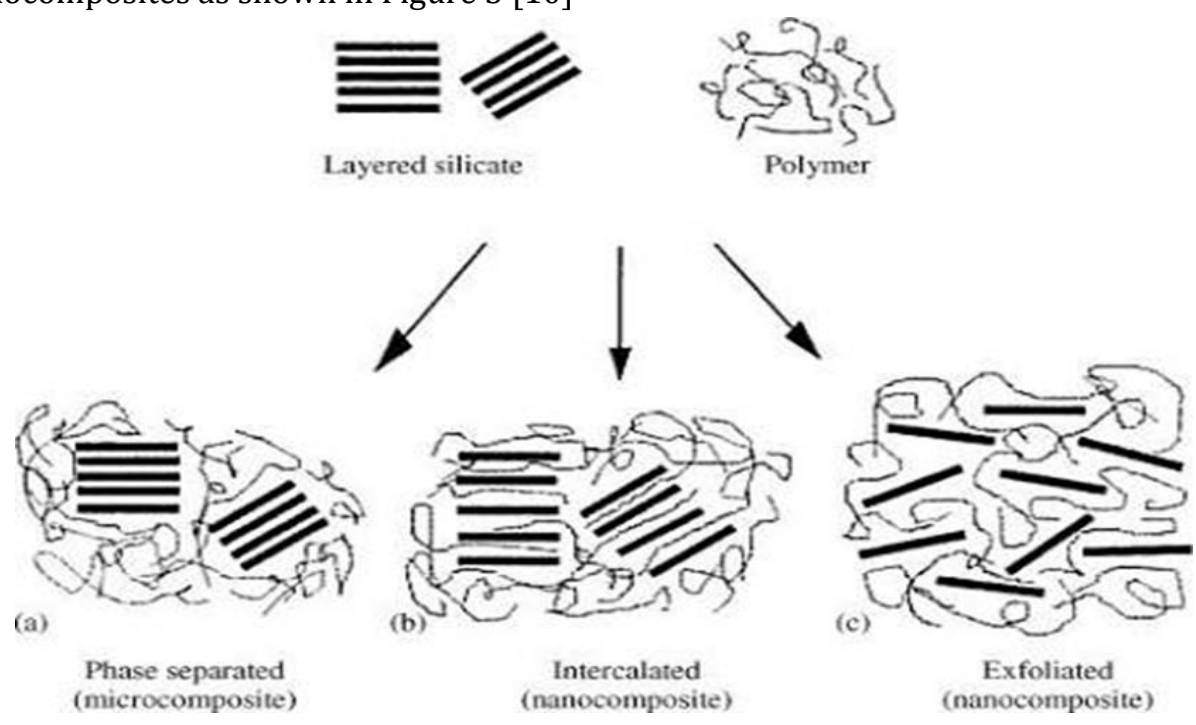

Figure 3 Three types of composite structures: (a) Phase separated microcomposite (b) Intercalated nanocomposite and (c) Exfoliated nanocomposite

\section{Pharmaceutical applications of nanoclay}

\subsection{Rheological modifier}

Rheological modifiers can control the flow properties of liquid systems such as paints, inks, emulsions or pigment suspensions by increasing the viscosity or impart thixotropic flow behavior to liquid system.

Also impart good color retention and coverage for nail lacquers, lipsticks and eye shadows. As organoclays are non irritant for both skin and eye contact therefore they are applicable as rheological modifiers in paints, inks, grease and cosmetics $[4,22,23]$.

\subsection{Particulate delivery systems based on clay minerals}

In particular, clay minerals can provide spontaneous submicron dispersions in aqueous media that result in low cost and biocompatible systems which is having a large surface area and high inclusion capacity. As the polymer/clay nanocomposites are a class of hybrid systems in which inorganic or organo- clay nanoparticles are dispersed in a polymer matrix. They have some interesting advantages as compared to the pure polymer, which may increase the mechanical as well as rheological properties and that may undergo good intercalation capacity offered by the clay mineral particles have been used to develop new controlled release systems [4, 24].

\subsection{Hydration- activated extended release systems}

Smectites successfully act as disintegrating agents in tablet formulations because of their hydrophilic and swelling properties that results by direct compression of sodium sulphathiazole and magnesium aluminum silicate of extended release tablets shows a progressive formation of a viscous gel layer around the tablets during in vitro dissolution tests [4].

\subsection{Improvement of dissolution rate by clay minerals}

As the improvement of dissolution rate of poorly water- soluble drugs remains one of the more important step for the pharmaceutical technologies. They may also undergo surface layer of adsorption. Molecules which are of finely divided solids greatly increase the surface area of the dissolution medium. Smectites were found to effectively increase the in vitro dissolution rate of non- ionic as well as acid insoluble drugs. Drug release from the clay surface is 
promoted by the weak bonding between them and due to the hydrophilic properties of the clay concomitantly drug wettability is enhanced. In comparison with phenytoin sodium capsules phenytoin- montmorillonite adsorbents are able to improve the bioavailability of the drug in humans [4, 25].

\subsection{Nanoclay as drug vehicle}

Nanoclay is potentially useful materials in the field of controlled release for the patients which shows the therapeutic activity and it acts as a drug vehicle. MMT could adsorb dietary toxins, bacterial toxins associated with gastrointestinal disturbance, hydrogen ions in acidosis and metabolic toxins such as steroidal metabolites associated with pregnancy. Among all these conditions the most common symptoms, including nausea, vomiting and diarrhea, most of which are typical symptoms of the side effects caused by anticancer drugs. A small addition of nanoclay can greatly increase the rheological properties. These properties are used in the treatment of pigment settling and sagging on vertical surfaces and gloss is minimum due to the low levels of addition. Nanoclay provides a good color retention as well as good coverage in cosmetics $[10,12]$.

Gene therapy is one of the most important treatment used for the genetic deficiencies and life- threatening diseases. The foreign DNA into cells, a carrier system is required thus recently, have been developed that has been successfully resulted that novel layered double hydroxide could form a nanohybrid by intercalating with bimolecular anion such as mononucleotides and thus DNA which shows the antisense oligonucleotide molecules packaged in a double layered hydroxide can enter cells by phagocytosis or endocytosis. The leukemia cells were used to explore the layered double hydroxides potential as gene carriers.

\subsection{Waste water treatment}

Nowadays the use of organoclays in waste water treatment has become common in industry. Organoclays can exhibit a synergistic effect with many commonly utilized water treatment unit processes including granular- activated charcoal, reverse osmosis, air strippers. Granular- activated carbon is very effective at removing a large range of organic molecules from water and very poor at removing large molecules such as humic acid and waste water containing emulsified oil and grease. Organoclays have proven to be the technology of choice for treating oily waste waters [12].

\section{Conclusion}

Use of polymer- clay nanocomposite in drug delivery system is an emerging area in the field of applications of nanotechnology. Besides this, another important application of these nanocomposites are in tissue engineering and in regeneration of nerve growth in a biological living system. Polymer- clay nanocomposites are a promising nano carrier available for the drug delivery of drug molecules. Polymer- clay nanocomposites used in oral multiparticulate system provide a slow and controlled drug release with improved mechanical integrity. Based upon the nanoparticle drug delivery it can provide the increased half-life, high biocompatibility, and minimum immunogenicity, site targeting and overcome the membrane barriers. Also the last era, major and new identifications have been significantly established in the smart material that alter its own structure and function in response to the environment. This performance has been used for the fabrication smart drug delivery systems, Smart polymer matrices release drugs by environment responses this system have been fruitfully achieved. In parallel the novel method of bottom-up and top-down nanofabrication technologies provided specifically controlled size and shaped nanoparticulate delivery system.

\section{Compliance with ethical standards}

\section{Acknowledgments}

The authors are thankful to Dr. L. Rathaiah, Chairman of Lavu educational society for providing necessary facilities to carry out the work.

\section{Disclosure of conflict of interest}

There is no conflict of interest. 


\section{References}

[1] Kamigaito 0. (1991). What can be improved by nanometer composites?. Journal of the Japan Society of Powder and Powder Metallurgy, 38(3), 315-321.

[2] Prajapati SK, Maurya SD, Das MK, Tilak VK, Verma KK and Dhakar RC. (2016). Dendrimers in drug delivery, diagnosis and therapy: basics and potential applications. Journal of Drug Delivery and Therapeutics, 6(1), 6792.

[3] Okpala CC. (2013). Nanocomposites-an overview. Int. J. Eng. Res. Dev, 8(11), 17.

[4] Suresh R, Borkar SN, Sawant VA, Shende VS and Dimble SK. (2010). Nanoclay drug delivery system. International Journal of Pharmaceutical Sciences and Nanotechnology, 3(2), 901-905.

[5] Vaia RA. (2002). Polymer nanocomposites open a new dimension for plastics and composites (No. AFRL-MLWP-TP-2005-408). Air force research lab wright-patterson AFB oh materials and manufacturing directorate.

[6] De Sousa Rodrigues LA, Figueiras A, Veiga F, de Freitas, RM, Nunes LCC, da Silva Filho EC and da Silva Leite CM. (2013). The systems containing clays and clay minerals from modified drug release: a review. Colloids and Surfaces B: Biointerfaces, 103, 642-651.

[7] Aguzzi C, Cerezo P, Viseras C and Caramella C. (2007). Use of clays as drug delivery systems: possibilities and limitations. Applied Clay Science, 36(1-3), 22-36.

[8] Iliescu RI, Andronescu E, Voicu G, Ficai A and Covaliu CI. (2011). Hybrid materials based on montmorillonite and citostatic drugs: Preparation and characterization. Applied Clay Science, 52(1-2), 62-68.

[9] Giannelis EP. (1996). Polymer layered silicate nanocomposites. Advanced materials, 8(1), 29-35.

[10] Patel HA, Somani RS, Bajaj HC and Jasra RV. (2006). Nanoclays for polymer nanocomposites, paints, inks, greases and cosmetics formulations, drug delivery vehicle and waste water treatment. Bulletin of Materials Science, 29(2), 133-145.

[11] Paul DR and Robeson LM. (2008). Polymer nanotechnology: nanocomposites. Polymer, 49(15), 3187-3204.

[12] Meng N, Zhou NL, Zhang SQ and Shen J. (2009). Controlled release and antibacterial activity chlorhexidine acetate (CA) intercalated in montmorillonite. International Journal of Pharmaceutics, 382(1-2), 45-49.

[13] Khalil, H, Mahajan D and Rafailovich M. (2005). Polymer-montmorillonite clay nanocomposites. Part 1: complexation of montmorillonite clay with a vinyl monomer. Polymer international, 54(2), 423-427.

[14] Wang X, Du Y and Luo J. (2008). Biopolymer/montmorillonite nanocomposite: preparation, drug-controlled release property and cytotoxicity. Nanotechnology, 19(6), 065707.

[15] Mohanambe L and Vasudevan S. (2005). Anionic clays containing anti-inflammatory drug molecules: Comparison of molecular dynamics simulation and measurements. The Journal of Physical Chemistry B, 109(32), 15651-15658.

[16] Pharmacognosy CK and Kokate SB. (2007). Gokhale Eds., Nirali Prakashan, Pune, India, 39th ed.

[17] Handbook of Pharmaceutical excipients, M.E. Quinn and R.C. Rowe Eds., Pharmaceutical press, USA, 6th ed., 2009.

[18] Joshi GV, Patel HA, Kevadiya BD and Bajaj HC. (2009). Montmorillonite intercalated with vitamin B1 as drug carrier. Applied Clay Science, 45(4), 248-253.

[19] Kevadiya BD, Thumbar RP, Rajput MM, Rajkumar S, Brambhatt H, Joshi GV and Bajaj HC. (2012). Montmorillonite/poly-( $\varepsilon$-caprolactone) composites as versatile layered material: Reservoirs for anticancer drug and controlled release property. European Journal of Pharmaceutical Sciences, 47(1), 265-272.

[20] Hua S, Yang $\mathrm{H}$ and Wang A. (2010). A pH-sensitive nanocomposite microsphere based on chitosan and montmorillonite with in vitro reduction of the burst release effect. Drug development and industrial pharmacy, 36(9), 1106-1114.

[21] Sharma R and Raina KK. (2011). Structural and Electrical studies on ferroelectric polymer nanocomposites (Doctoral dissertation).

[22] Choy JH, Choi SJ, Oh JM and Park T. (2007). Clay minerals and layered double hydroxides for novel biological applications. Applied Clay Science, 36(1-3), 122-132. 
[23] Forest MG, Zhou R and Wang Q. (2004). Chaotic Boundaries of Nematic Polymers in Mixed Shear and ExtensionalFlows. Physical Review Letters, 93(8), 088301.

[24] Nicholson BF, Clancy P and Rick SW. (2006). The interface response function and melting point of the prism interface of ice Ih using a fluctuating charge model (TIP4P-FQ). Journal of crystal growth, 293(1), 78-85.

[25] Armstrong G and Kailas L. (2017). Hyphenated analytical techniques for materials characterization. European Journal of Physics, 38(5), 053001.

\section{How to cite this article}

Paravastu VKK, Yarraguntla SR and Suvvari A. (2019). Role of nanocomposites in drug delivery. GSC Biological and Pharmaceutical Sciences, 8(3), 94-103. 